\title{
Simple Protocol for NMR Analysis of the Enantiomeric Purity of Primary
}

\section{Amines}

Yolanda Pérez-Fuertes, Andrew M. Kelly, Andrew L. Johnson, Susumu Arimori,

Steven D. Bull ${ }^{\star}$ and Tony D. James.*

In the version published February 9, 2006, page S7 of the Supporting Information inadvertently contained the 1H NMR spectra of a substrate not included in Table 1 of the manuscript. This resulted in subsequent compounds on pages S8-S11 being incorrectly labeled with reference to Table 1 of the manuscript. The corrected version was published March 21, 2006. 
${ }^{1} \mathrm{H}$ NMR Spectra of Complexes of Table 1

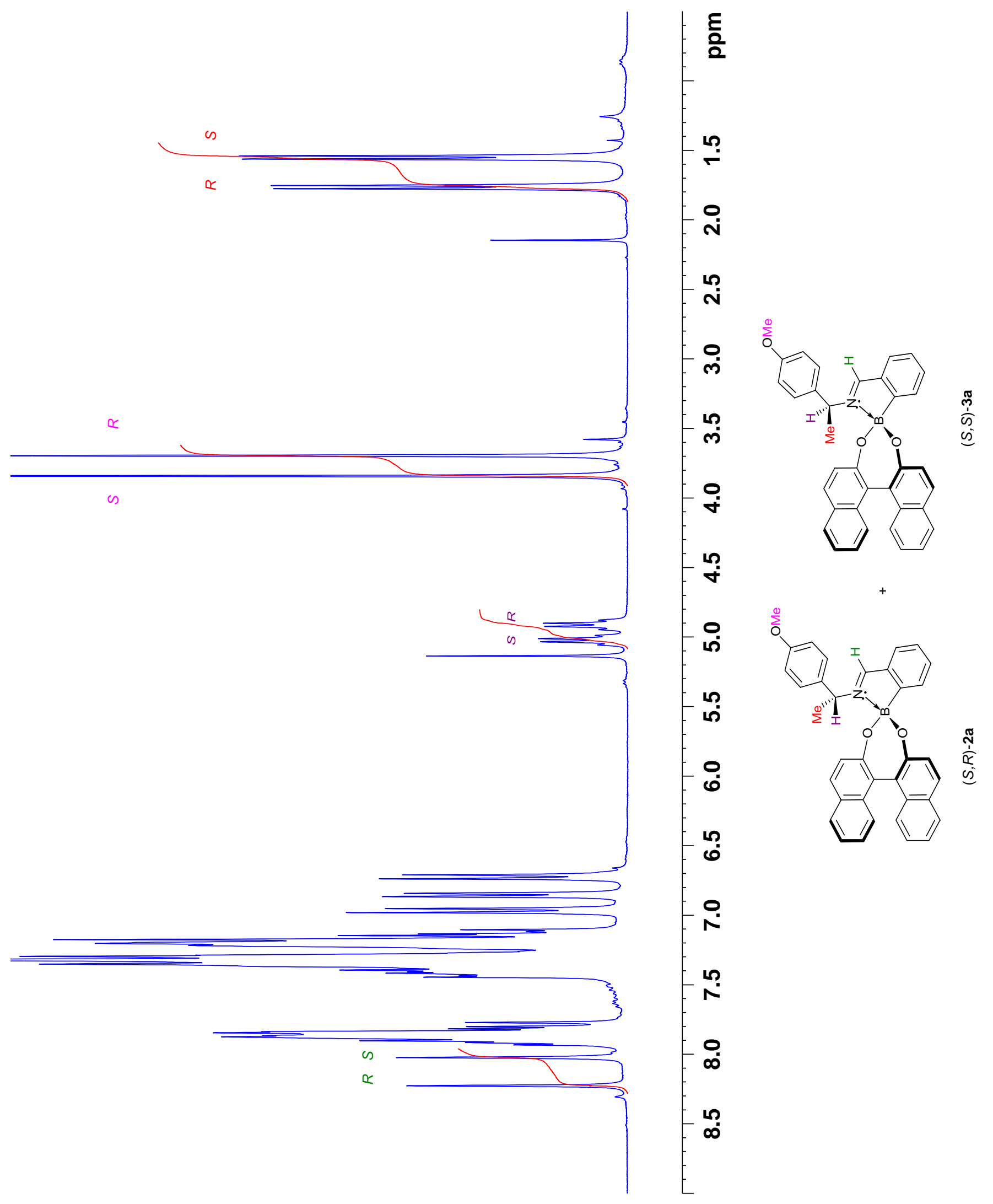




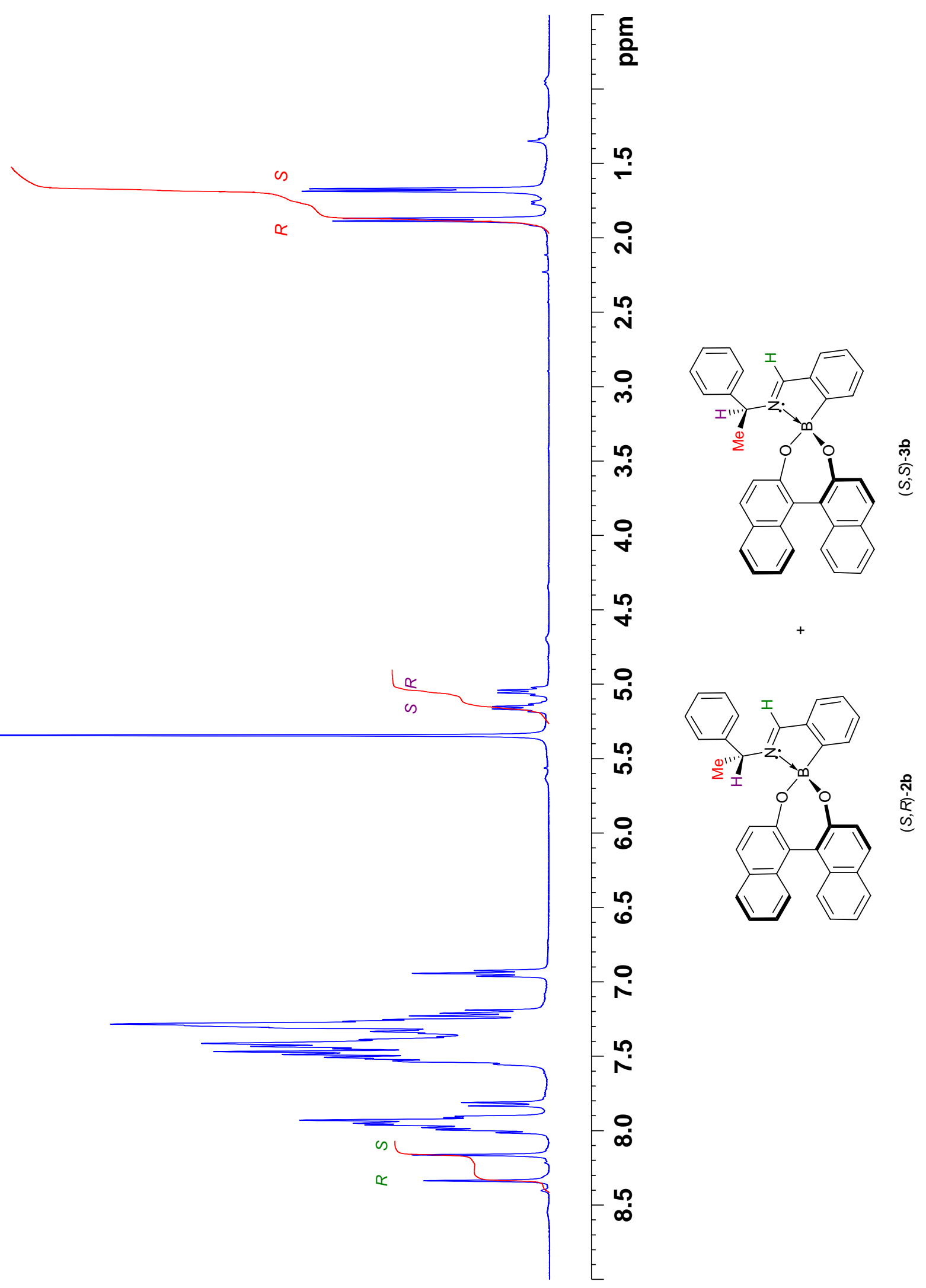




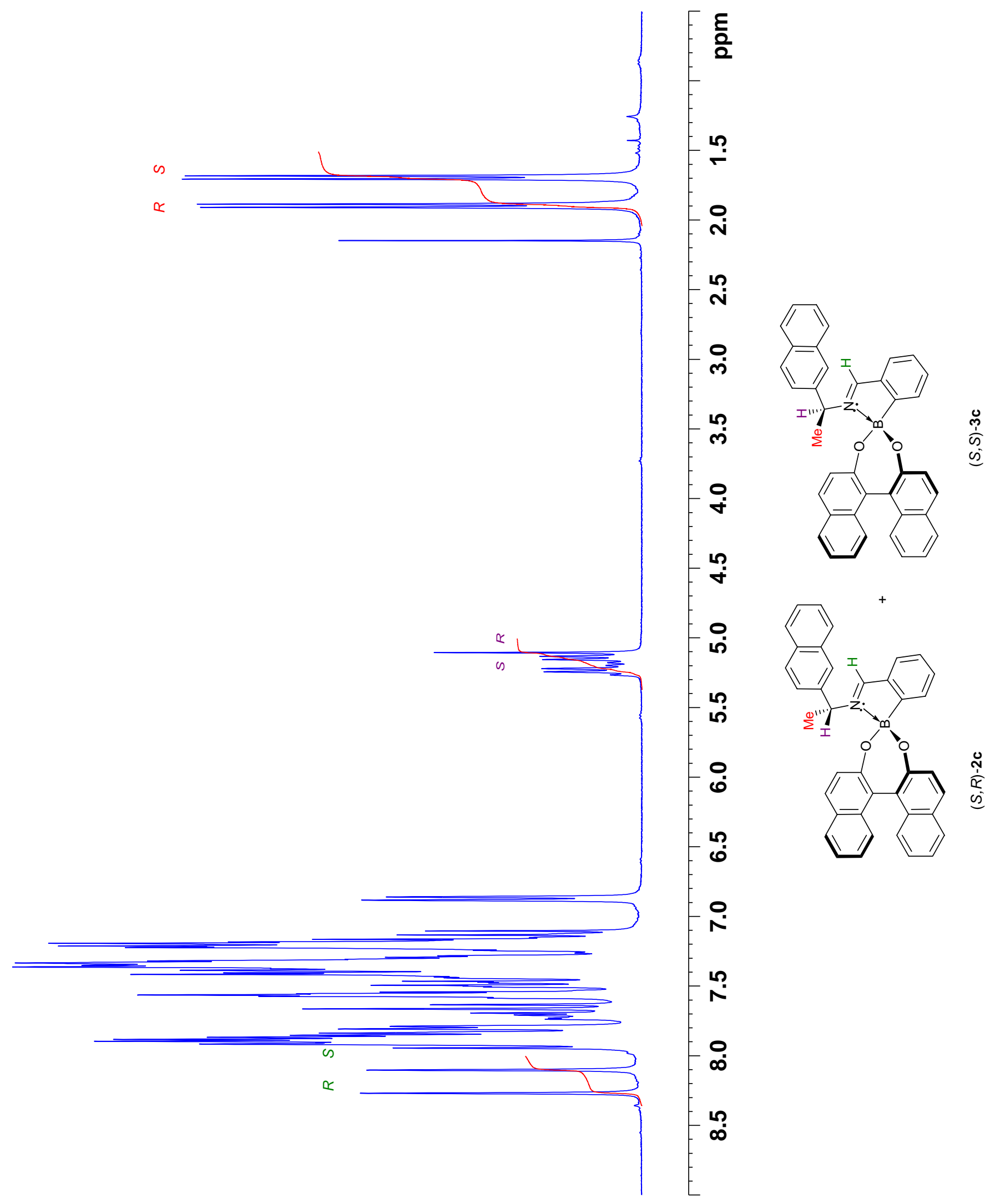




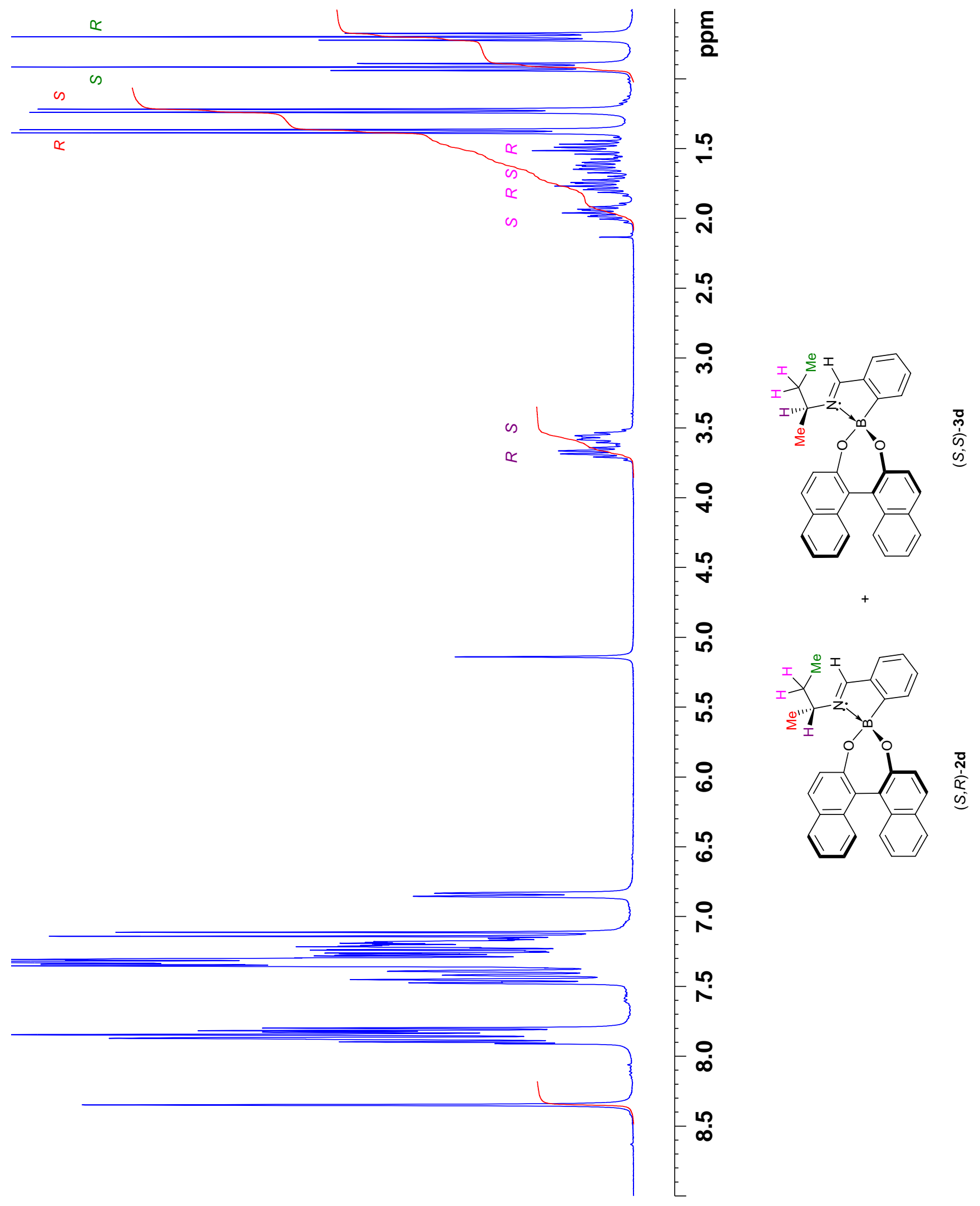




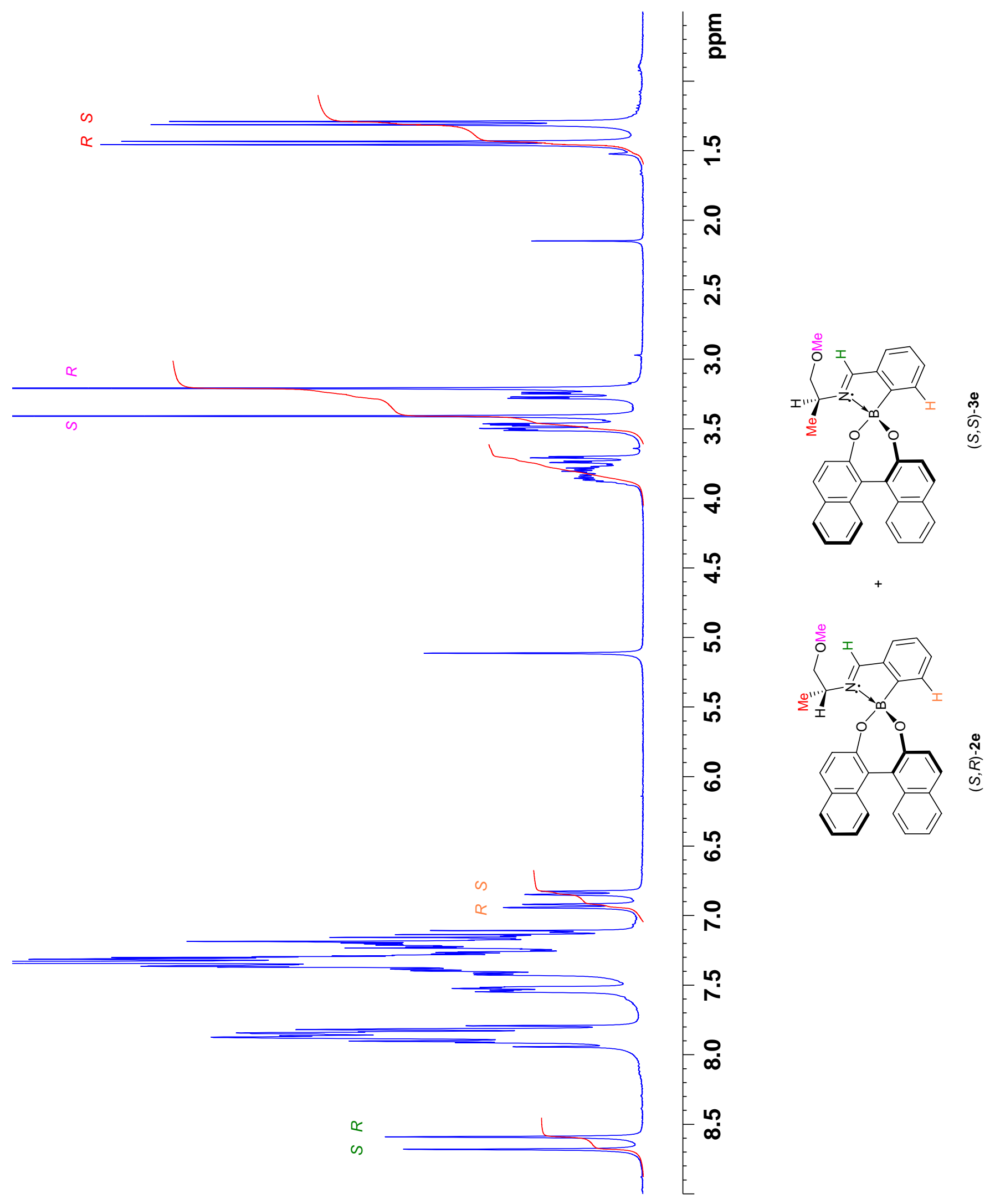




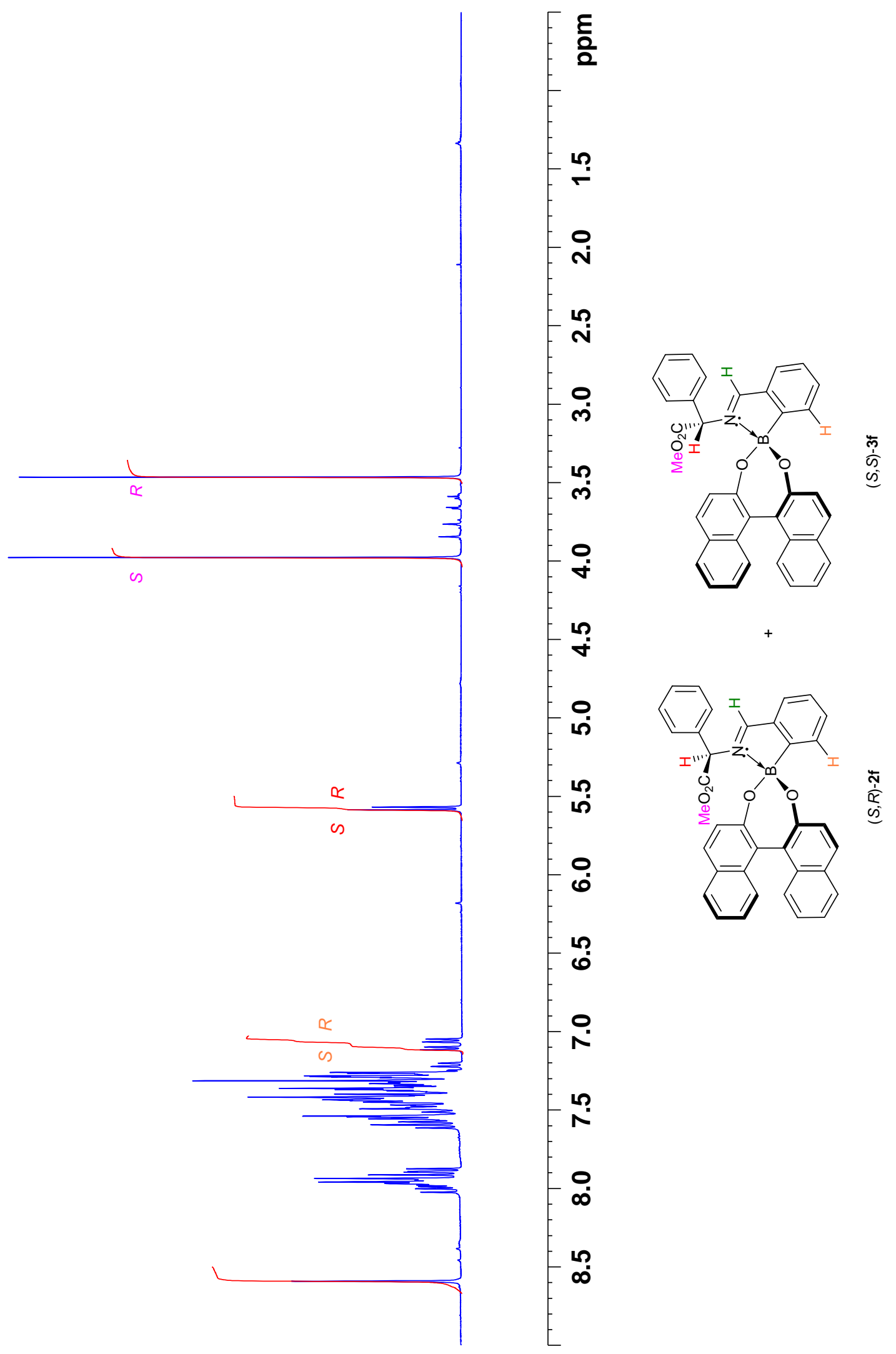




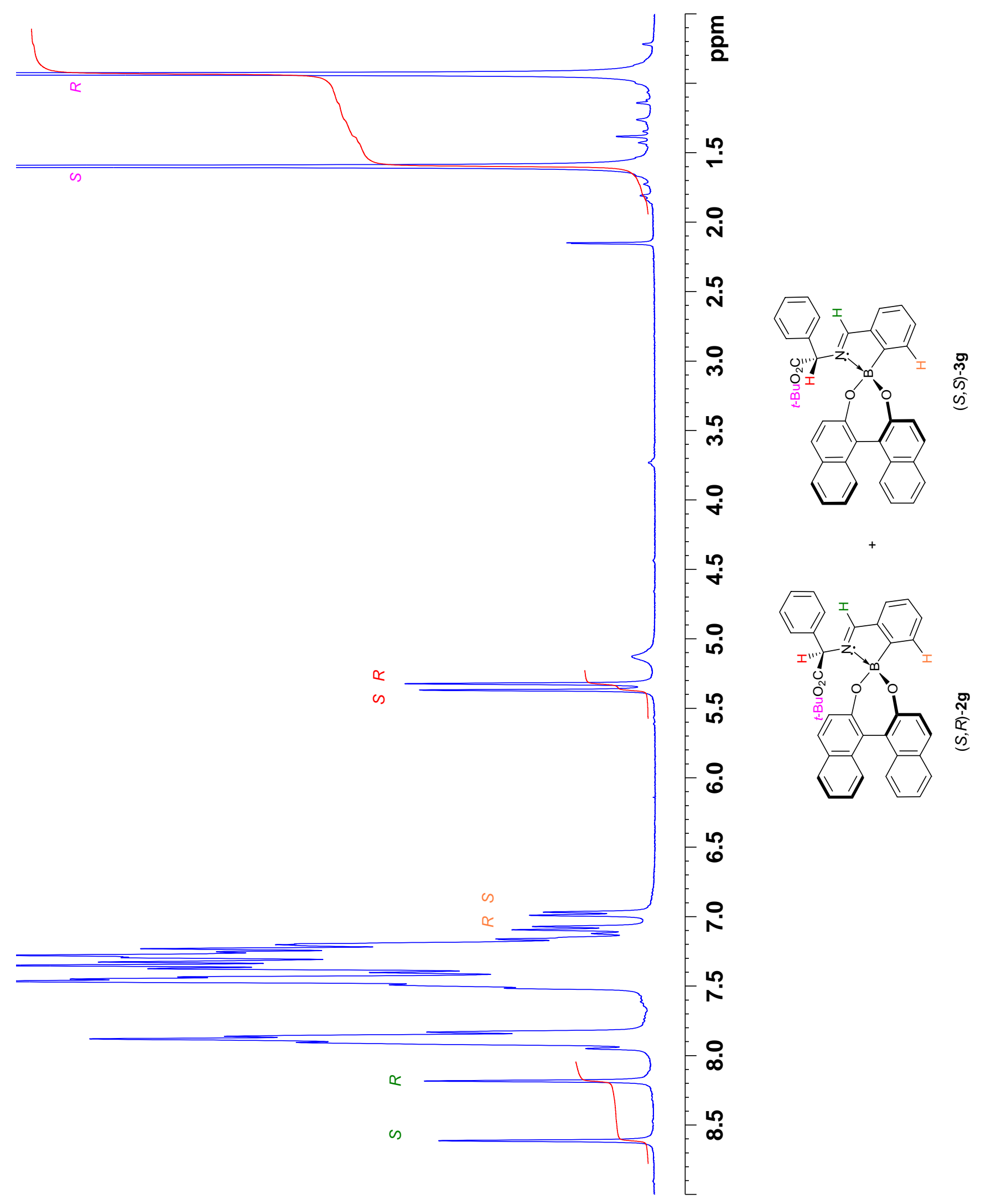




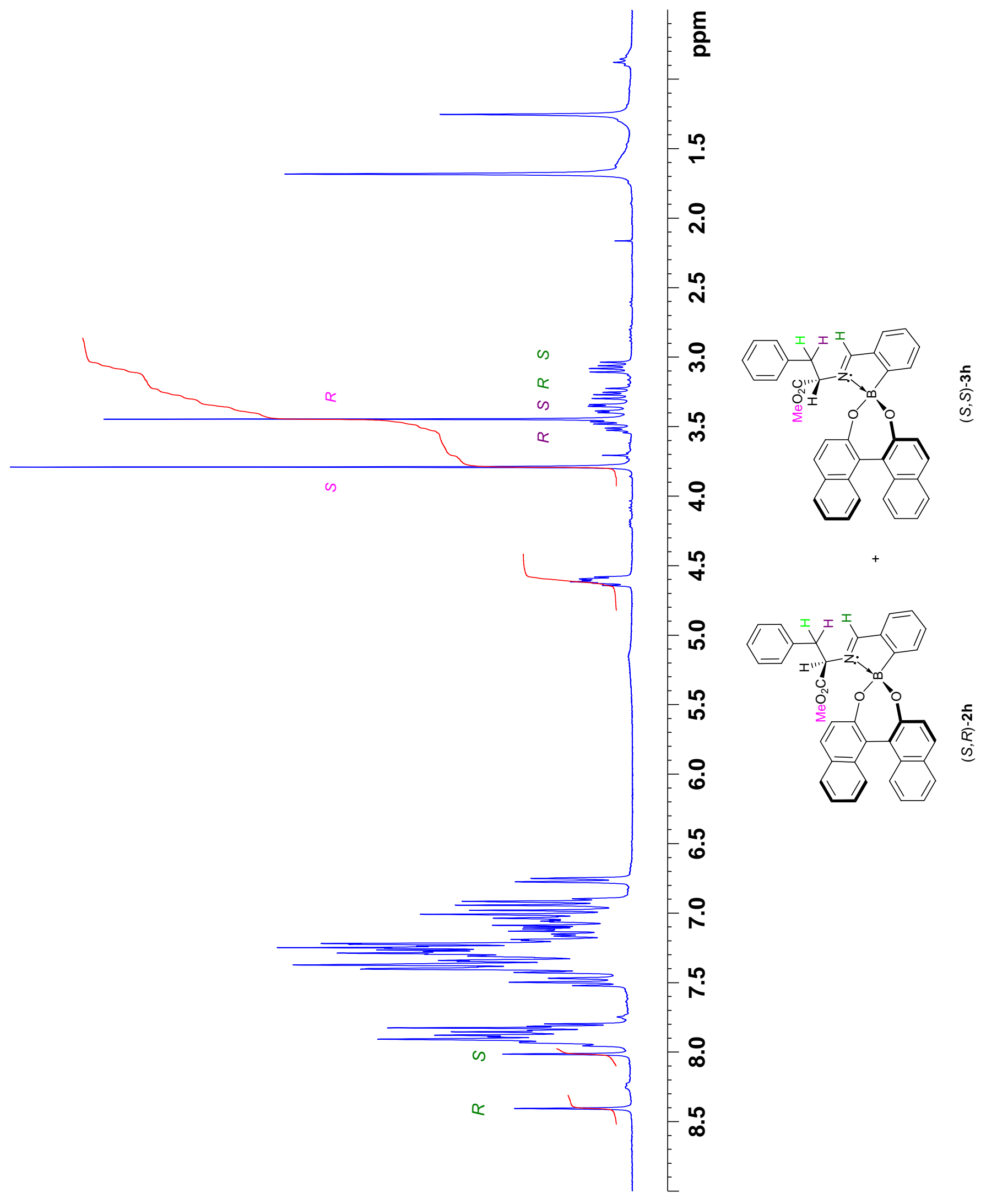




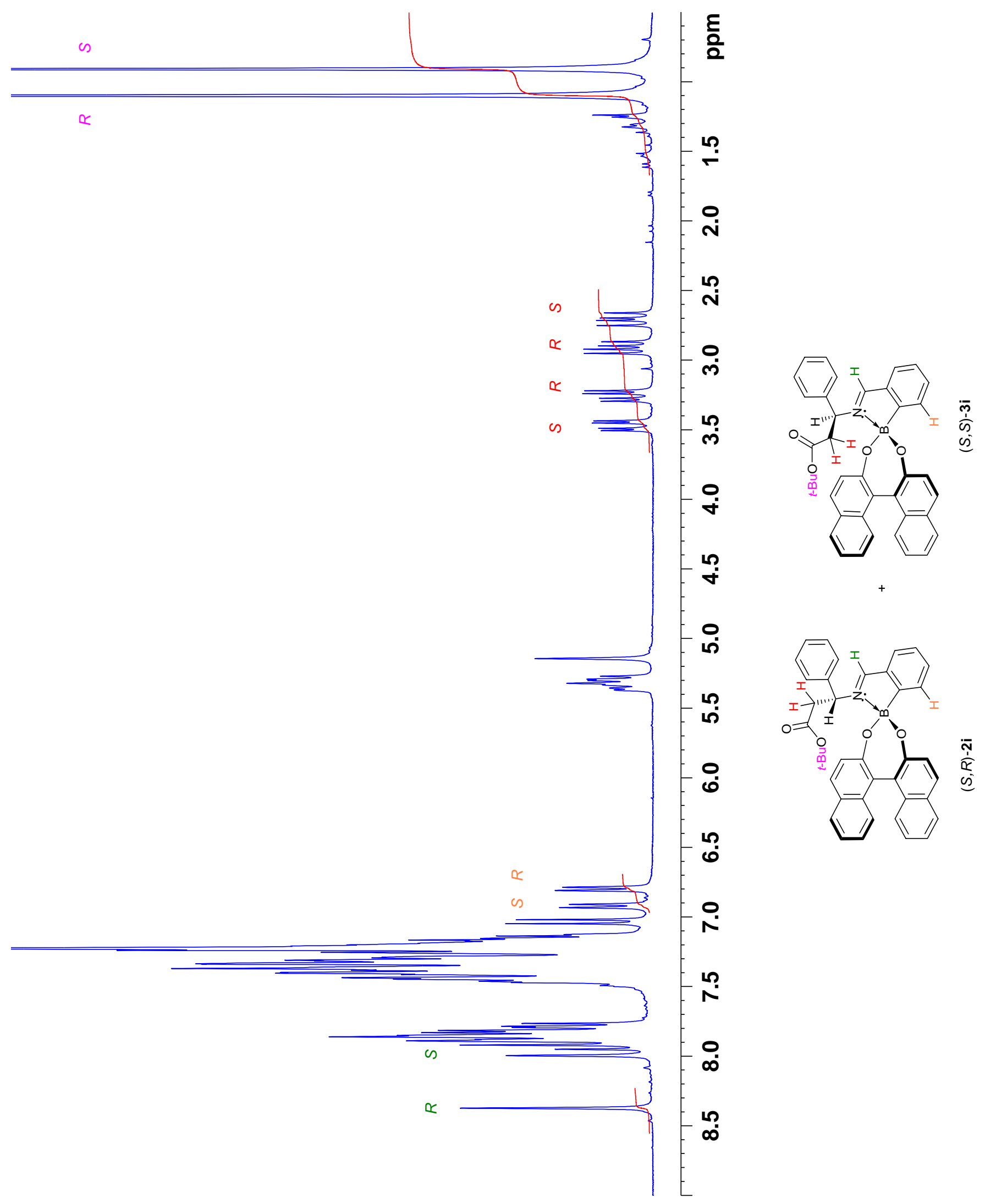


${ }^{1}$ H NMR Spectra of Complexes of Table 2

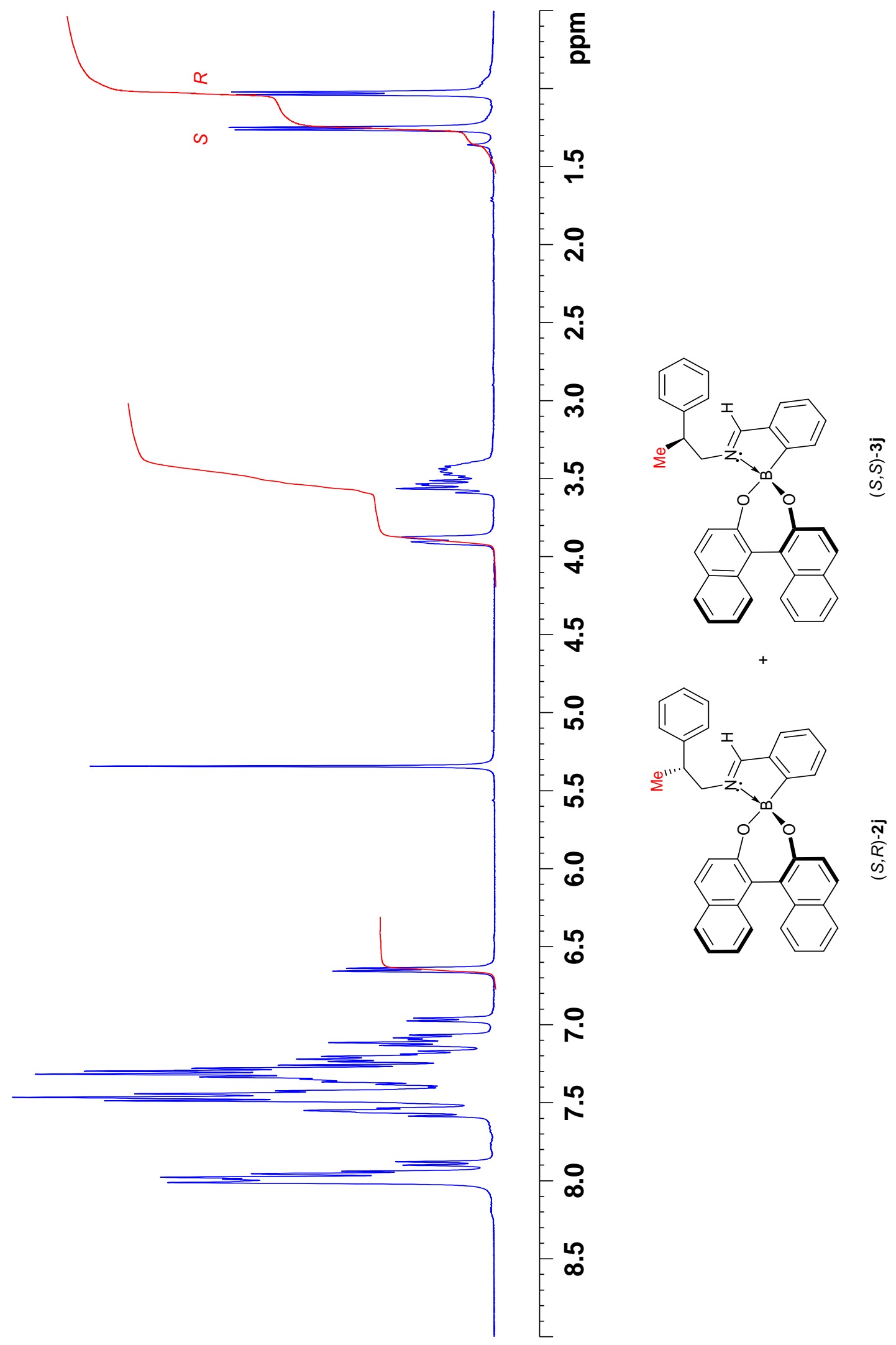




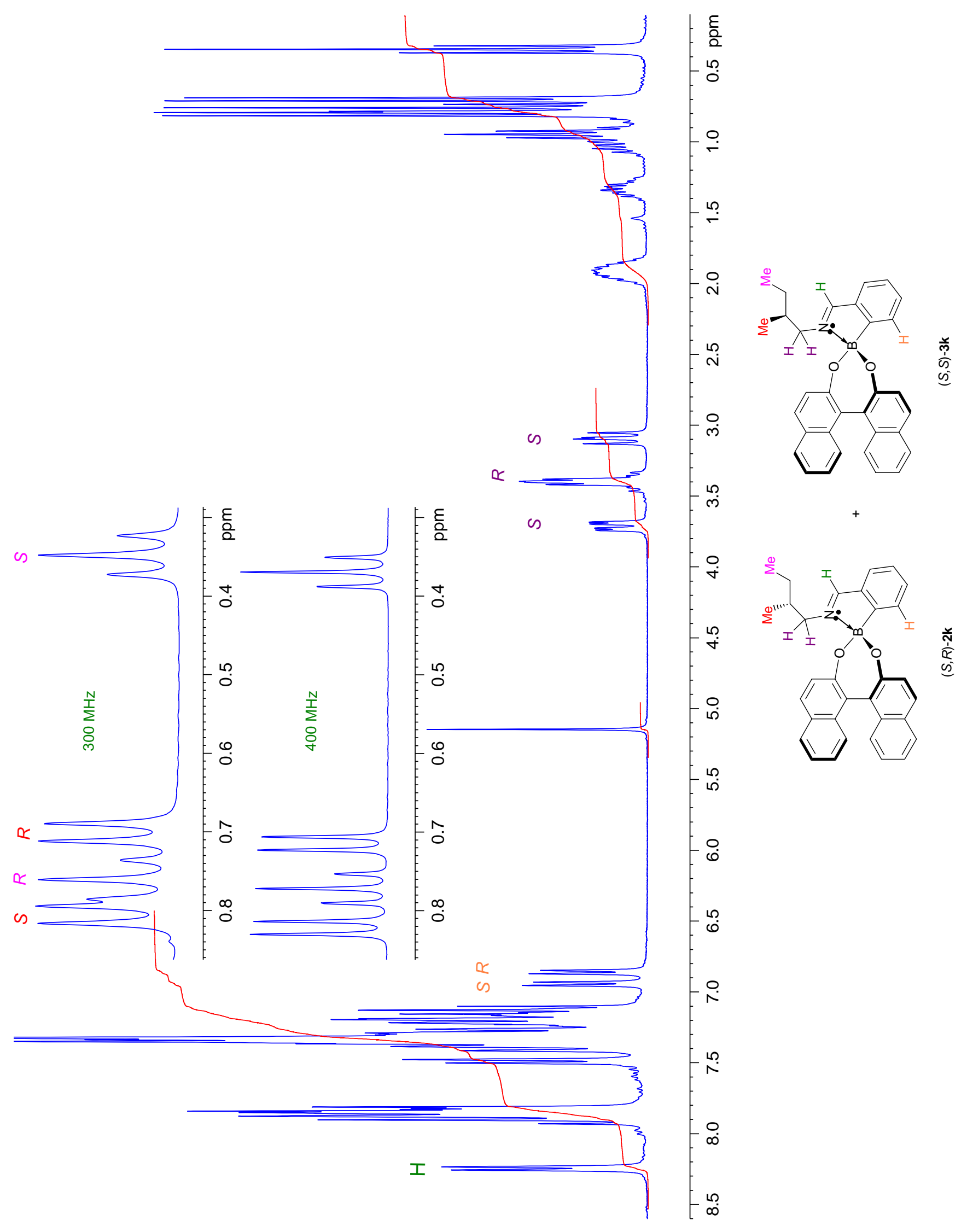




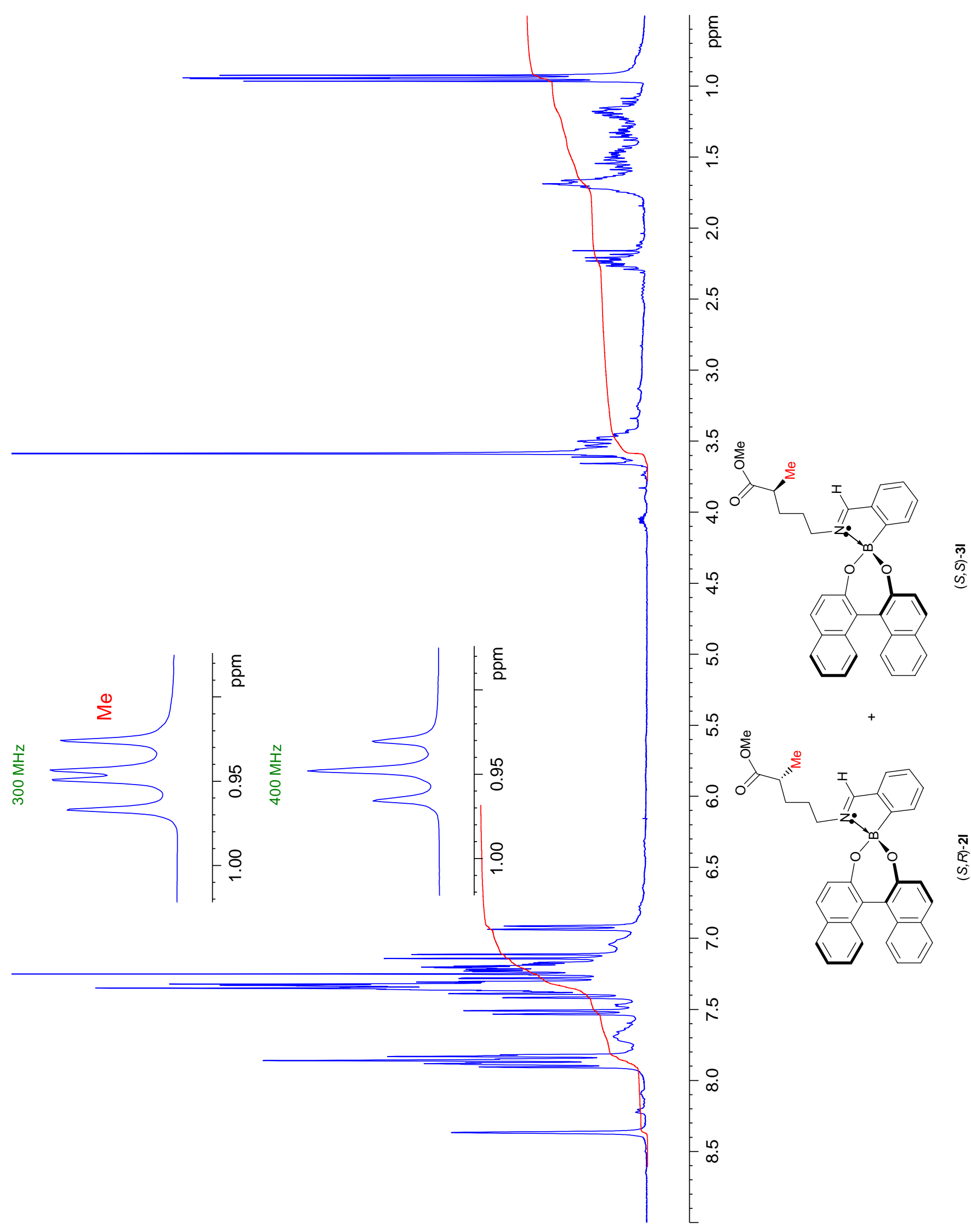




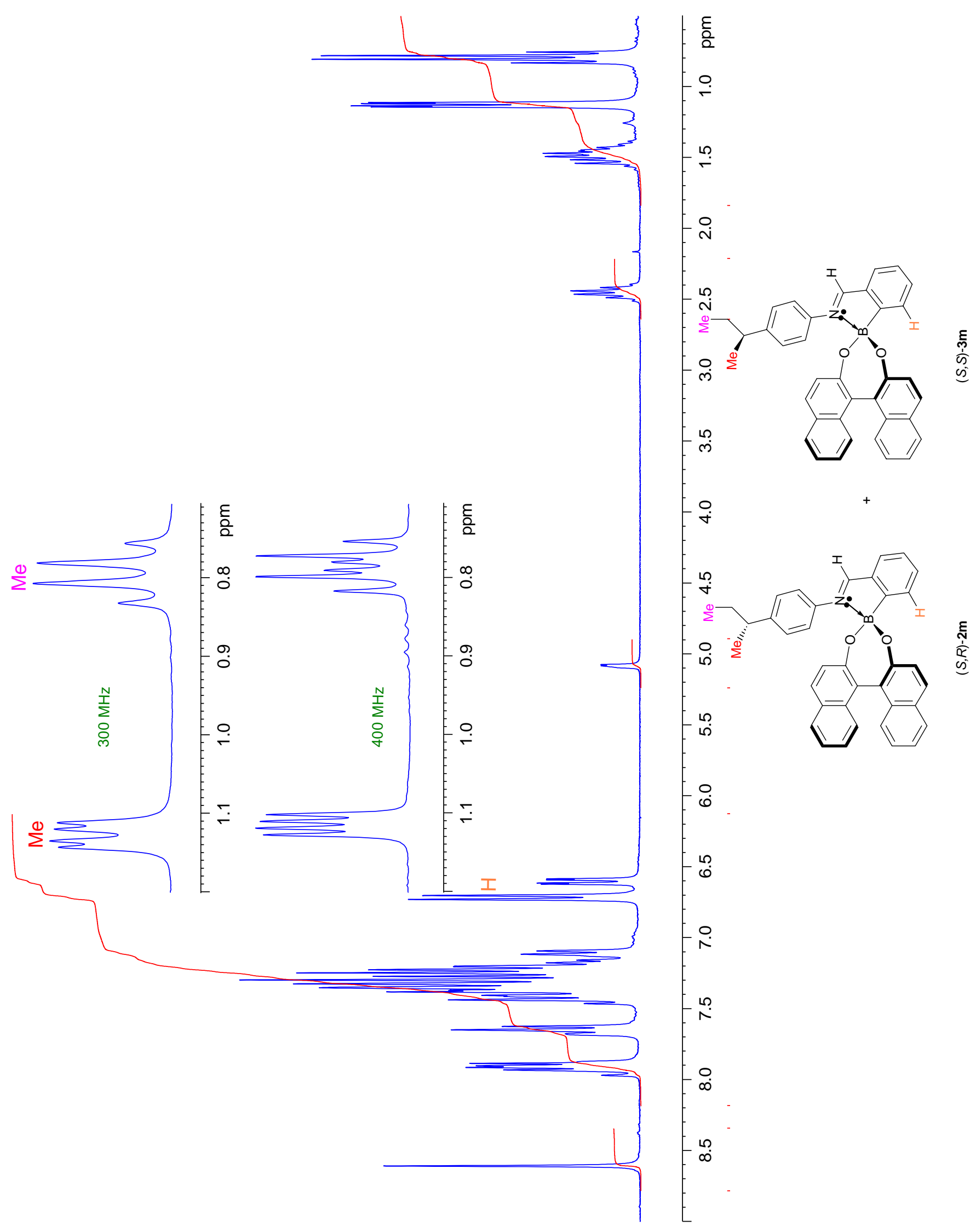

